\title{
La inteligencia emocional del gestor educativo en tiempos de pandemia
}

\author{
Fecha de recepción : 2021-03-30 • Fecha de aceptación: 2021-06-07 • Fecha de publicación: 2021-09-10
}

Ramón Antonio Hernández Chirinos de Jesus ${ }^{1}$ Universidade Estadual do Piauí, Brasil ramon_hernandez2012@hotmail.com https://orcid.org/0000-0002-0663-8983

Francisco das Chagas Silva de Jesus Hernández ${ }^{2}$ Secretaria Municipal de Educación do Piauí, Brasil Professordjesus.2013@yahoo.com.br https://orcid.org/0000-0002-5420-0679

\section{Resumen}

La inteligencia emocional permite generar en cada ser humano la forma de balancear sus propios sentimientos, y el de los demás. En este escenario pandémico, el gestor educativo ha aprendido a controlar su inteligencia emocional de manera que pueda dar cuenta de su gestión. El presente artículo tuvo como propósito principal estudiar la inteligencia emocional del gestor educativo en tiempos de pandemia. Para ello, se realizó una investigación de carácter cualitativo y documental, donde a través de diversos materiales bibliográficos, se elaboró el texto, para luego emitir la opinión de los autores sobre el tema abordado. Se determinó que la inteligencia emocional en los gestores educativos generó cambios significativos en el desempeño de sus funciones y en las relaciones con las personas a su cargo, lo que permitió tener un autoaprendizaje que repercutió positivamente en su ámbito laboral y social.

Palabras clave: inteligencia emocional, gestión, pandemia, gestor educativo. 


\begin{abstract}
Emotional intelligence allows to generate in each human being the way to balance his own feelings, and those of others. In this pandemic scenario, the educational manager has learned to control his emotional intelligence so that he can account for his management. The main purpose of this article was to study the emotional intelligence of the educational manager in times of pandemic. For this purpose, a qualitative and documentary research was carried out, where through various bibliographic materials, the text was elaborated, to then issue the opinion of the authors on the topic addressed. It was determined that emotional intelligence in educational managers generated significant changes in the performance of their functions and in their relationships with the people in their charge, which allowed them to have a self-learning that had a positive impact on their work and social environment.
\end{abstract}

Keywords: emotional intelligence, management, pandemic, educational manager. 


\section{Introducción}

En la actualidad, la inteligencia emocional (IE) se ha convertido en un elemento clave dentro de las instituciones educativas. Ya que las emociones juegan un papel primordial en el proceso de enseñanza y aprendizaje, donde es necesario formar al ser humano en el desarrollo de sus habilidades emocionales, afectivas y sociales.

Por otro lado, existen emociones negativas que pueden afectar la gestión escolar y la práctica docente, lo cual genera dificultades, miedos e inseguridad, de tal manera que puede ocasionar conflictos en las relaciones interpersonales, así como también un ineficiente desarrollo de las funciones establecidas, lo que impide mantener un adecuado clima organizacional y de gestión.

Sin embargo, el factor emocional siempre ha estado latente en todas las organizaciones, pues las mismas son indispensables para decidir las determinaciones correctas o el control sobre ellas, y de esa manera, poder lograr los objetivos, ejecutando las funciones en el ámbito educativo. En este sentido, la inteligencia emocional prevalece sobre el razonamiento y accionar del gestor educativo.

Es oportuno decir que, los desafíos de la educación de hoy día son cada vez más relevantes, provocando en los gestores y educadores mucha inquietud. Por ello, al poner en práctica la inteligencia emocional en la escuela, permitirá que los estudiantes y profesores tengan un mayor manejo sobre sus aspectos emocionales, porque a pesar de todo, las personas siempre tienen pensamientos positivos y negativos, momentos de dificultad y sentimientos que les molestan.

Por lo tanto, es elemental aprender a enfrentar las emociones, controlando el comportamiento y moldeando las actitudes de forma positiva para el beneficio propio. El desarrollo de esta debe estar presente en el seno familiar, para luego ser reforzado en el niño cuando acuda a la institución educativa. Así, no solo se aprenderá los contenidos educativos, sino que será capaz de diferenciar algunas características y habilidades emocionales. Entonces, sí el estudiante desenvuelve la capacidad de lidiar con sus emociones, las mismas serán de gran ayuda para su desempeño académico y su futuro profesional.

De hecho, el modelo de inteligencia emocional de Goleman (2008) propone una serie de habilidades básicas tales como la percepción, la asimilación, comprensión y regulación emocional, las cuales, una vez desarrolladas estas habilidades en el gestor, se puede verter su radio de acción hacia los docentes, dado que, al reconocer y colocar en práctica sus capacidades cognoscitivas, y afectivas en el contexto, logrará un mejor desempeño profesional.

Es relevante decir que, el escenario pandémico que se vive en el mundo, ha permitido que las instituciones educativas se vean obligadas a aceptar de forma urgente las políticas, estrategias y normas, que les permitiesen poder darle continuidad a la educación de cientos de niños, jóvenes, y adultos, quienes se vieron afectados por la suspensión de las clases presenciales. Lo cual les obligó a recorrer una educación virtual, que, en la mayoría de los casos, desafortunadamente solo benefició a cierto sector de la población brasileña. 
Vale la pena señalar que, cada año escolar que inicia, los gestores educativos encaran en sus escuelas una serie de nudos críticos que les exigen tomar decisiones asertivas que contribuyan a minimizar los riesgos administrativos y pedagógicos. Estas amenazas pueden convertirse en verdaderas tragedias hasta el punto de poner en peligro la vida de los estudiantes, familias y profesionales de la educación. Es por ello que, en estos tiempos de pandemia, los gestores educativos han puesto en práctica su inteligencia emocional, de tal manera que han podido establecer y adoptar de forma exitosa medidas sanitarias en el recinto educativo, en tal caso; el uso de máscaras y protectores faciales, higiene frecuente de las manos, preservación de la distancia social, adecuada ventilación de aulas, organización de entrada y salida, evaluación de la temperatura en la entrada del recinto, además de la elaboración de un plan de trabajo para la limpieza y la desinfección periódica de las instalaciones de la escuela, de manera que, se pueda garantizar un espacio que sea seguro y agradable para todos.

En medio de la pandemia, la gestión educativa se apoyó de la inteligencia emocional, originando mudanzas considerables que le permiten al director tener una visión desafiante ante lo que será su administración. Es por ello que, los líderes escolares debieron reinventarse en este tiempo de salubridad pública. Pues aprovechándose del momento coyuntural, lograron hacer un llamado de alerta a las personas que conforman la comunidad educativa, para que valorasen su vida como algo esencial. Esta reinvención permitió que la escuela pasara por un proceso de transformación que no perjudicara a ningún miembro de su comunidad, pero sí que les permita tener un crecimiento sustancial en todo lo que se propongan.

A pesar del cierre de las escuelas como medida preventiva para evitar la propagación a gran escala del nuevo coronavirus, el gestor escolar, haciendo uso de su inteligencia emocional, siempre permaneció en su sitio de trabajo, tratando de mediar y prever todas las posibles consecuencias. En este caso hubo falta de profesores y otros miembros del personal; dificultad en el suministro de materiales para uso administrativo, pedagógicos, entre otros.

Con base a lo anterior, hay que resaltar el trabajo incansable de los gestores educativos, quienes han tenido que cambiar su ritmo de trabajo, adaptándose a las diferentes leyes emergenciales creadas por el Gobierno de estado, en donde los municipios deben reajustarse a dichos decretos. Para contextualizar lo que se vive, se dieron diferentes cambios de forma drástica en las instituciones educativas, lo cual trajo como consecuencia que los gestores educativos se readapten a una nueva forma de analizar, pensar, reflexionar y actuar, todo con el propósito de mantener un clima emocionalmente estable entre sus empleados. En este caso, el gestor debe mantener un equilibrio emocional dentro y fuera de la sala de clase, lo cual se convierte en equidad, bajo un ambiente propicio, lleno de aspectos positivos y creativos, donde sus subordinados se comprometan y ejerzan un trabajo armonioso que enriquezca el proceso enseñanza y aprendizaje de los educandos (Goleman, 2008, p. 18).

\subsection{Inteligencia emocional}

Cuando se habla de inteligencia emocional se hace referencia al nombre que se le da al conjunto de habilidades relacionadas con el manejo de las emociones, especialmente en momentos de estrés que ayudan a percibir, procesar, comprender y saber maniobrar situaciones de angustia y 
crisis. Por lo general el ser humano tiende a sobrepasar el espacio, desplegando la capacidad de formar, regir y vigilar sus actos mentales y las actividades de información. Pues bien, se educa, se explora, se relaciona y mantiene una balanza entre tantas cosas, sin saber cuáles son las situaciones mentales que se pudiesen vincular.

Para Goleman (2014, p. 20), es posible definir la inteligencia emocional de la siguiente manera: "capacidad que incluye autocontrol, celo y persistencia, así como la capacidad de motivarnos". Se puede observar que el autor presenta una definición que valora y centraliza emociones de las personas, destacando que se basa en el autocontrol, la perseverancia y capacidad que tiene cada ser humano para motivarse, e incluso para aquellos que se definen como pesimistas, tristes o depresivos. Lo que quiere decir que, cualquier persona tiene dentro de sí, los elementos necesarios para marcar la diferencia, además de, permitir que todo su aprendizaje esté dirigido a su campo profesional, así como a sus logros personales.

En este sentido se deja entrever la posibilidad de incluir en la base nacional curricular de todos los niveles de la educación brasileña, trabajar con el estudiantado la inteligencia emocional, ya que, cada estudiante es un ser único, y como tal, tiene sus propias emociones, que cuando su motivación es incentivada, le facilita al docente un mejor desarrollo en el proceso de aprendizaje, así como una mejora en las relaciones e interacción entre el facilitador y el educando, dentro y fuera de la comunidad escolar.

Según Goleman (2014, p.22), se debe "infundir desde la niñez las competencias humanas como: la seguridad, el dominio de sí mismo, la capacidad de ponerse en el lugar del otro, y el arte de escuchar (...) podrá solucionar los enfrentamientos”. Inspirar y trabajar estas emociones con los niños les proporcionarán las bases para un aprendizaje consciente, centrado en el significado de conceptos e ideas, puesto que, en el interior de cada uno de ellos, hay una fuente inagotable de emociones positivas que le abren el camino al disfrute de un aprendizaje marcado bajo diferentes entornos que desarrollarán sus habilidades, las cuales estarán presentes durante cada etapa de su vida.

La inteligencia emocional determina el potencial para aprender los fundamentos de autocontrol y cosas por el estilo, la competencia emocional muestra el nivel de dominio de ese potencial, el cual puede traducirse en habilidades profesionales. Lo que quiere decir que, el aprendizaje basado en la inteligencia emocional, permite que se sienta ese potencial para aprender las concepciones básicas del autodominio, y desde esa perspectiva, el individuo logra transformarlos en aprendizajes y habilidades profesionales (Goleman, 2014, p. 15).

Retomando lo dicho por el autor, no hay forma de negar el papel fundamental de las emociones en la vida de los seres humanos, todo individuo actúa a partir de sus emociones, en este sentido, cada uno de ellos debe analizar y reflexionar sobre la importancia de nutrir sus emociones desde un ámbito positivo, es decir, que se tenga el control sobre ellas, para tomar sus propias decisiones.

Actualmente las organizaciones educativas se encuentran en cambios constantes, donde se está actualizando y capacitando a todo su personal sobre el tema de la inteligencia emocional, ya que puede representar la clave para alcanzar el éxito, al momento de gerenciar con eficacia, logrando 
convertirse en un gestor con competencias para ser aplicadas en la comunidad estudiantil (López, 2016, p. 24)

\subsection{Los cinco pilares de la inteligencia emocional}

\section{- Autoconciencia}

Representa la capacidad de conocerse a sí mismo, es el paso inicial para comprender y poner en práctica la inteligencia emocional, siendo el principal conocimiento que dependerá del interés de cada ser humano, por lo tanto, nadie puede asumir emocionalmente el lugar del otro. La autoconciencia le permite al hombre poder reconocer sus sentimientos y emociones, en especial en aquellos momentos cuando se enfrenta a una emoción fuerte. Entonces, la autoconciencia se muestra como un proceso de autorreflexión, que coloca al individuo en una etapa consciente de lo que está sintiendo, e incluso puede permitirle estar ausente o posponer la toma de decisiones impulsadas por tales emociones.

\section{- Autocontrol o gestión de las emociones}

En el transcurrir de la historia del mundo, el equilibrio emocional, la prudencia y la moderación de la exageración se han convertidos en los signos de sabiduría y calidad en el comportamiento de las personas. Para Goleman (2011), el objetivo es el equilibrio y no la supresión de emociones: cada sentimiento tiene su valor y su significado. Una vida sin entusiasmo sería como vivir en un desesperante desierto de neutralidad, aislado de las bellezas de la vida. Y aunque el individuo puede tener una actitud equilibrada de sus emociones, se valora, el desarrollo de ese equilibrio, el cual no siempre se ha visto como algo necesario para aprender, sino que, muchas veces se ve como algo natural, es decir, la gente se entrega a sus sentimientos y emociones, donde pasa su vida siendo dirigido por ellos, cometiendo los mismos errores y actitudes que obstaculizan su desarrollo personal y profesional, sin permitirse la autoconciencia, de manera que pueda buscar el cambio en este escenario.

Goleman (2011) también subraya que las emociones no se pueden controlar, pero es su duración la que marcará la diferencia entre sentir un estado negativo y deshacerse de él lo antes posible, o quedarse alimentando este sentimiento. La vida de las personas está plagada constantemente por la presencia de conflictos, a veces menos o más, y especialmente en la vida profesional, la gente siempre está enfrentando las turbulencias antes que los problemas, y la gestión de estas emociones en este momento es de vital importancia para mantener la concentración y la motivación en el proceso que se está desarrollando. Y aunque no se puede evitar las emociones de ira, indignación, desánimo, las cuales pueden ser transitorios, siempre y cuando el ser humano esté consciente de cómo ellas lo afectan y cómo pueden afrontarlas. Porque la permanencia de estos sentimientos negativos puede, de cualquier forma, perjudicar la salud psicológica y física de la persona.

\section{- Automotivación}


Se ha entendido que solo las personas motivadas podrían realizar sus actividades de forma más completa. Y mucho más que los factores externos, la automotivación es la principal energía que influye para mantener la atención en los logros. Goleman (2011) sostiene que una de las mayores dificultades es mantenerse motivado, es decir, no desmotivarse ante los fracasos de la vida. Es aquí, donde la inteligencia emocional recobra su verdadero significado, porque el sujeto que se enfrenta a momentos estresantes y turbulentos, sí no posee conocimiento de sus emociones y de qué manera estas pudiesen influir en sus determinaciones, logrará rendirse ante la ansiedad y desesperanza, tomando decisiones equivocas que lo alejará del camino cierto para alcanzar sus logros.

\section{- Empatía}

Representa la capacidad de ponerse en el lugar del otro, mostrando la sensibilidad ante diversas situaciones. Con la empatía se busca la comprensión de los sentimientos que van más allá de la comunicación verbal, porque las personas no siempre expresan lo que sienten con palabras, sino con gestos y expresiones faciales. Por ello, se requiere que el otro esté en sintonía con sus propias emociones, de manera que pueda interpretar estas demostraciones con habilidad.

Los sentimientos de empatía se convierten en particularidades que permiten un acercamiento en las relaciones personales y profesionales. En las instituciones, las personas empáticas se ganan la credibilidad de los demás, creando un ambiente de seguridad que les ofrece confianza para hablar de lo que realmente sienten y de lo que experimentan en sus actividades. De este modo, la conexión emocional se centra en el compartir y en la buena comunión, donde todos se benefician, prosperan, se desarrollan y crecen. Así, esta interrelación logra que las personas sean proclives a nuevos conocimientos y estén abiertas a la búsqueda de soluciones que favorezcan a todos y no a un pequeño grupo de ellos (Cooper \& Sawaf, 2004, p.79).

\section{- El arte de vivir en sociedad}

Influir y contagiar a los demás son habilidades pertinentes que forman parte del arte de vivir en sociedad, es decir, que el ser humano no pasa por la vida de los demás de forma desapercibida, sino que, de alguna manera deja su huella o contribución. El arte social es uno de los factores más emblemáticos de la inteligencia emocional, ya que, el alto grado de inteligencia cognitiva no garantiza la certeza de vivir en el entorno social. Si embargo, no se puede descartar que la misma está vinculada directamente con la capacidad de autocontrol y empatía del sujeto inteligentemente emocional.

\subsection{La inteligencia emocional en estudiantes y profesores}

Para el contexto educativo actual, es propicio citar la Base Nacional Comum Curricular (BNCC) (2019), que propone la educación socioemocional como una de sus 10 competencias, cuyo fin es desarrollar la inteligencia emocional en estudiantes y profesores. Para ello, la escuela y sus gestores educativos necesitan estar emocionalmente preparados para saber cómo fomentar estas habilidades entre el personal estudiantil, académico, administrativo y obrero. 


\section{- Estudiantes}

Dado que el desarrollo emocional tiene una fuerte influencia en este contexto, las instituciones educativas deben jugar un papel decisivo en la enseñanza de niños y jóvenes. Para esto se puede apoyar en las disciplinas que de una u otra manera trabajen las emociones, porque lo que se quiere es que los socios de aprendizajes se animen a compartir sus experiencias y sentimientos. Pues es a través de la educación emocional que se pueden vincular actividades pedagógicas con todas las áreas del conocimiento. Para que esto acontezca todo debe ser coordinado por los mediadores de aprendizajes, ya que es un proceso un poco más complejo. Sin embargo, se puede generar momentos de debate menos artificiales, problematizando las relaciones y emociones que surgen durante el trabajo en equipo o cuando un alumno se siente molesto por sus profesores o compañeros.

\section{- Profesores}

El papel del docente en el aprendizaje social y emocional es fundamental, ya que necesita poner en práctica su sensibilidad para abrir el debate y ofrecer un espacio de expresión adecuado para los estudiantes. El profesional de la docencia debe actuar con la intención de preparar a sus educandos para que estos sean conscientes y responsables en su forma de sentir, pensar y actuar. Por eso es imprescindible que el mediador de aprendizajes esté preparado para comprender todo lo que involucra la inteligencia emocional. Es difícil desarrollar estas particularidades en otros, pero se puede lograr a través de una formación que demuestre la importancia de estas características para los docentes. Porque hasta ahora, nadie sabe cómo tener inteligencia emocional, pero la misma experiencia puede servir para dar pautas sobre cómo desarrollar esa habilidad.

Por ejemplo, cuando la inteligencia emocional es puesta en práctica en el recinto educativo, este hecho permite que los estudiantes y docentes puedan controlar sus hábitos, costumbres, conocimientos y limitaciones, con el fin de identificar los aspectos que necesitan ser mejorados. Entonces, en medio de este escenario pandémico, el gestor educativo debe elegir cuáles pudiesen ser las herramientas que promuevan el desarrollo social, emocional y psicológico de los estudiantes y docentes.

\section{- Gestión escolar}

El tema de la gestión escolar surgió de la necesidad de interpretar la correlación que se instaura dentro de los centros educacionales, así como los retos y posibilidades que enfrentan sus gestores. Dado que, la gestión escolar se ha encaminado a nuevos horizontes en el transcurrir de los años. Entonces se ha tenido que adoptar a una novedosa forma de gestión escolar en la que se establece el control democrático como un estandarte de lucha, en el enfrentamiento de las desigualdades sociales y económicas. En consecuencia, se puede observar cómo la gestión escolar en conjunto con la organización docente sigue un mismo norte que busca apropiarse de una estructuración completa, que garantice una educación de calidad. Es importante enfatizar que la gestión escolar tiene como función dirigir las dinámicas culturales de una escuela, junto con las políticas públicas educativas, y los lineamientos para la implementación de un proyecto pedagógico institucional, además, debe comprometerse con los principios de la democracia y los 
métodos que propicien las condiciones para un ambiente educativo autónomo, dando soluciones propias, en el escenario de sus competencias.

Teniendo en cuenta la perspectiva de formación y seguimiento de los gestores escolares en los institutos educacionales, a ellos se les debe permitir ser un agente de cambio, capaces de generar las acciones que aseguren el cumplimiento de una gestión que sea integral, democrática y participativa. Según Machado (1999) en Machado (2000, p.102), en el contexto de los cambios que invadieron el escenario educativo actual y la gestión escolar, la educación continua ha ido ganando importancia progresiva como signo de que el aprendizaje debe ser asumido desde un carácter permanente y dinámico, que forme parte vivencial los profesionales. Pues los mismos deben desarrollar sus competencias, que involucren valores, conocimientos y habilidades para afrontar los cambios acelerados, en contextos complejos, diversos y desiguales. También se cree que estas habilidades pueden contribuir con la consolidación de un nuevo modelo de gestión educativa, que permita que la educación brasileña pueda implementar cambios necesarios para la consecución de una educación de calidad, de acuerdo con las exigencias de la sociedad moderna.

Se debe tener en cuenta que la gestión escolar debe cooperar desde un espacio que se centre en la calidad educativa, pues su principal objetivo debe promover la organización, movilización y articulación de todas las condiciones materiales y humanas esenciales para asegurar el progreso de los recintos educativos. Siendo coparticipe en el aprendizaje efectivo que adquieran los estudiantes, de forma que, les permita asumir y enfrentar los diversos retos que les aguarda en un mundo tan complejo.

Por su parte, Luck (2010) señala que al asumir la gestión de una escuela, el gestor educativo tiene el compromiso de ser competente en el entorno laboral, estableciendo su autonomía, pero al mismo tiempo, aceptando la participación de los actores en el contexto escolar, donde existe un trabajo colectivo, pero también compartido, además de la consecución de objetivos comunes.

\subsection{Formación de los gestores escolares}

Hoy día la formación de los gestores escolares debería estar a cargo de las diversas universidades, pues ellas son las responsables de ofrecer al estudiantado sus primeras nociones sobre la función que debe ejercer un gestor. Por lo general, ese profesional de la docencia debe haber egresado de cualquier carrera humanística independientemente del área de conocimiento. Esta condición cobrará relevancia sobre las acciones que el gestor realice en la construcción, implementación y evaluación del proyecto pedagógico que debe tener la unidad escolar (Gracindo, 2009, pág. 141). Por lo tanto, el gestor educacional debe verse como un educador, y no solo como un gerente de procesos organizacionales. Donde su autoridad se legitime no tanto por sus habilidades en el manejo de gestión como tal, sino por su perfil profesional en la educación, siendo capaz de liderar diversas situaciones presentes en su entorno laboral (Estêvão, 2001, p. 99).

Al estar consciente de los enunciados que definen la gestión escolar, de una u otra manera se puede contribuir con el quehacer docente en todas sus dimensiones. Por tal razón, Fiorentini (2005) afirma que en la práctica curricular se da la interacción entre los mediadores y estudiantes, 
pero todo dependerá de la organización y gestión de la enseñanza, donde cada docente establezca el espacio-tiempo y actividades didácticas con sus educandos" (p. 108).

Por su parte, Gracindo (2009) es partidario de la idea de que el docente es el gerente y cuentadante en el aula, y afirma que "es posible que todo profesor esté en la capacidad de ser el interlocutor al participar en la gestión democrática que se desarrolla en la escuela, como facilitador de conocimientos o gestor educativo" (p. 142).

Desde el momento en que este mundo pasó a ser globalizado, se vio en la necesidad de construir un espacio para los procesos de enseñanza y aprendizaje, en donde se empezó a valorar más de cerca la gestión escolar, ya no con la figura del director rígido que se dedicaba exclusivamente a aspectos administrativos de la institución, sino como la persona que gerencia, aprende, retroalimenta y recibe observaciones para crecer cada día.

\subsection{Impactos psicológicos negativos de Covid-19 en la gestión escolar}

La acelerada proliferación del coronavirus por todo el mundo, la inseguridad sobre cómo controlar la gravedad del Covid-19, así como lo imprevisible sobre el tiempo que este pudiese durar, además de, las posibles consecuencias relacionadas con la salud mental, convirtiéndose en factores de riesgo para la ciudadanía en general, además de la exageración de algunos medios de comunicación, pudieran ser los causantes de las agonías psicológicas de los profesionales de la gestión escolar, pues esta situación única les pudiese generar momentos donde tenga que experimentar sentimientos de sufrimiento, preocupación o malestar, en vista de lo que pasó a ser su nueva forma de vida.

Estas pruebas originan percepciones de que algo no está en su lugar, o que en cualquier momento ocurra un evento traumático que afecte las acciones de las personas. Es desde allí, cuando se empieza a indagar sobre los estados emocionales de estudiantes y docentes que enfrentan las clases de una enseñanza remota o completamente a distancia.

Según Miguel (2020), la señal clave para que las actividades educativas sean reorganizadas va a depender de los impactos emocionales producidos por medidas de aislamiento social en el aprendizaje de los estudiantes. Pues se debe considerar la suspensión indefinida de las actividades presencialmente en los ambientes escolares.

Todo esto ha desatado diversos elementos emocionales de frustración y ansiedad por parte del profesorado y gestores de los recintos educativos. Para ello, se aconseja que los gestores escolares, en colaboración con la institución educativa, investiguen la forma de como minimizar el miedo, la tristeza y la frustración de los miembros de la comunidad estudiantil.

Sin embargo, es de vital importancia que el director institucional consiga la participación más activa en el recinto educativo, actuando de manera intensa, continua y eficaz en la búsqueda de una escuela independiente, participativa, transformadora, pero principalmente se comprometa con educación de calidad que esté encaminada a las nuevas políticas públicas. La gestión escolar debe estar guiada por fundamentos de responsabilidad con la enseñanza. Pues estos elementos 
deben llevar al gestor a la reflexión sobre el ambiente pedagógico, para que, de esa manera, puedan construir una gestión participativa y no autocrática.

Sin embargo, los gestores no solo deben limitarse al aspecto administrativo de una escuela, sino poder contribuir con gestión pedagógica, considerada como eje principal, en el que el directivo debe ser el gerente de enseñanza y aprendizaje. Para que el centro educativo logre los modelos de calidad en la producción de conocimiento, es necesario que el gestor se convierta en un articulador activo y participativo en todos los proyectos relacionados con el campo formativo de su escuela. Se sabe que la gestión pedagógica es la base fundamental de una escuela, porque una institución bien administrada, desarrolla calidad docente a través de la planificación, seguimiento y la evaluación de la propuesta pedagógica; seguida del desempeño de los educandos, mediadores de aprendizaje y todas las personas que integran su equipo gestor.

\section{Metodología}

El enfoque metodológico utilizado en el estudio se centró en la investigación cualitativa, siendo de carácter documental, pues los resultados que soportan este artículo se limitan a las distintas conclusiones que respaldan sus bases, derivados de los artículos y diversos medios electrónicos mundiales. De igual manera, se consideraron otras publicaciones que enfatizan la inteligencia emocional del gestor escolar, su relación con el personal docente, administrativo y obrero en tiempos de pandemia.

En el mismo orden de ideas, el estudio se desarrolló bajo el enfoque cualitativo, que de acuerdo con Sánchez (2019), explica que "la investigación cuyo enfoque sea cualitativo se sustenta en evidencias que se enfocan hacia la especificación detallada del fenómeno con la intención de comprenderlo y explicarlo" (p. 102). Para tal caso, el fenómeno que se relaciona con el objeto de estudio es la inteligencia emocional del gestor educativo en tiempos de pandemia.

En este proceso de indagación se utilizó el diseño documental, que según Bernal (2010), es donde se hace un análisis de la información escrita sobre un determinado tema, con el objetivo de construir nexos, diferencias, etapas, actitudes o comparaciones entre lo ya acostumbrado y lo actual del conocimiento, respecto al tema que se pretende estudiar. De igual forma, el mismo autor establece cuáles son las principales fuentes documentales, entre ellas están: documentos escritos (libros, periódicos, revistas, actas notariales, tratados, conferencias escritas, páginas web, textos on-line, entre otros), documentos fílmicos (películas, diapositivas y videos), también documentos grabados; en ese sentido, se investigó en diferentes referencias documentales, las cuales permitieron recabar los insumos de relevancia para el estudiar la inteligencia emocional de los gestores educativos en tiempos de pandemia.

\subsection{Procedimiento de la investigación}

Después de una selección en profundidad de documentos se escogieron estrategias específicas, utilizando la técnica de Bardin, a través de los siguientes pasos: pre-análisis, exploración del material y la obtención de los resultados con el uso de la inferencia e interpretación; determinando 
el eje temático que fue abordado y contemplado de manera consistente, lo que permitió identificar resultados satisfactorios de la recogida.

Para Bardin (2010), el análisis de contenido se caracteriza por ser un conjunto de técnicas de análisis en el campo de las comunicaciones que pretende comprender desde la sistematización de datos, contenido de mensajes. Por lo tanto, se utilizó el análisis de contenido temático para categorizar los datos obtenidos en la exploración inicial de los documentos. Luego, se procedió a emitir una conclusión, con base a lo analizado e interpretado en los hallazgos, para de esta forma comunicar las ideas relevantes de los autores.

\section{Conclusiones}

La inteligencia emocional representa el conjunto de habilidades que implican los diferentes tipos de emociones; que se usa generalmente para reconocer el significado de las emociones y sus relaciones al momento de resolver nudos críticos. Cuando cada ser humano aprende a maniobrar su inteligencia emocional, será capaz de reconocer, percibir y valorar las propias emociones para autorregularlas y expresarlas en los momentos más pertinentes. El propósito de la inteligencia emocional reside en la habilidad para reconocer, percibir y valorar las propias emociones, así como para regularlas y expresarlas en los momentos apropiados y en las formas pertinentes. Término utilizado por Goleman (2014), donde la define como: "la capacidad que tiene cada ser humano de reconocer sus propios sentimientos y los ajenos, de motivarlos para que haya un buen manejo de las emociones, en todas las personas".

El propósito primordial de esta investigación fue estudiar la inteligencia emocional del gestor educativo en tiempos de pandemia. De manera que pueda ser capaz de reconocer y manejar sus emociones, logrando, así relacionarse con los demás, de forma adecuada y pacífica, porque tan solo el hecho de que el gestor educativo aplique la inteligencia emocional en su entorno laboral, le podrá garantizar una mejor relación con el personal a su cargo.

En este momento de cambios drásticos, por causas de la pandemia, la gestión escolar debe poner en práctica la inteligencia emocional, permitiéndoles establecer un clima laboral armónico, estimulador en el trabajo ejecutado por los profesionales de la educación, respetando la autoestima y sus buenas relacionales interpersonales con su equipo docente, administrativo y obrero, pues la mayoría de ellos se encuentran en situaciones emocionales diversas a causa de lo vivido por la pandemia. En otras palabras, el gestor debe ser empático cuando su personal experimente sentimientos de emociones negativas como rabia, ira, tristeza e incluso depresión por el aislamiento social y falta de recursos económicos, que no les permite tener calidad de vida.

Es oportuno mencionar que la IE ha hecho contribuciones relevantes a esta investigación, tanto así que, se pudo ver el cambio significativo en el desempeño de los gestores escolares, en especial durante los momentos de incertidumbre causados por el Covid-19. Por otro lado, a través de este estudio se logró obtener información valiosa sobre la forma en cómo los gestores educativos se vieron afectados ante este inesperado acontecimiento, el cual les permitió trabajar su inteligencia emocional de modo que la pandemia no influyese negativamente sobre la comunidad educativa, ya 
que al principio provocó una profunda tristeza, confusión e inseguridad en los padres, profesores y hasta en los propios estudiantes.

Sobre la base de las conclusiones expuestas, este trabajo defiende la postura de incorporar la IE en la vida de los profesores, estudiantes, familias y en especial en los gestores escolares, quienes, a pesar de la suspensión de las clases presenciales, dieron continuidad a los trabajos pedagógicos con mucha energía y disposición, de tal modo que, esa energía fuese transmitida a todos los educandos, para así mantenerse motivados a pesar de todas las situaciones acarreados por la pandemia. Se puede concluir que, por triste y difícil que haya sido este período pandémico, los gestores educativos, con apoyo del personal docente, administrativo y obrero, no renunciaron a su misión, pues la educación debe seguir avanzando de manera que garantice la inclusión de niños, jóvenes y adolescentes en un sistema educativo lleno de desigualdades. 


\section{Referencias}

Bardin, L. (2010). Análisis de contenido. Ediciones 70.

Bernal, C. (2010). Metodología de la Investigación, administración, economía, humanidades y ciencias sociales. Editorial Pearson Educación

Base Nacional Comum Curricular. (2019). Educação é a base. http://basenacionalcomum.mec.gov.br/

Cooper, R., \& Sawaf, C. Y. (2004). La inteligencia emocional aplicada al liderazgo ya las organizaciones. Editorial Norma.

Estêvão, C. A. V. (2001). Gestão educacional e formação. Revista Gestão em ação, 87-105. http://www.gestaoemacao.ufba.br/revistas/gav4n201.PDF\#page=87

Fiorentini, D. (2005). A formação matemática e didático-pedagógica nas disciplinas da licenciatura em matemática. Revista de Educação PUC-Campinas, (18), 107-115. https://periodicos.puc-campinas.edu.br/seer/ index.php/reveducacao/article/view/266/2945

Goleman, D. (2008). Inteligencia emocional em las empresas. Editorial Kairos.

Goleman, D. (2011). Inteligencia Emocional: A Teoria Revolucionaria Que Redefine O Que E Ser Inteligente. Editora Objetiva.

Goleman, D. (2014). Inteligencia emocional. Editorial Kairos. Temas e Debates e Círculos de Leitores.

Gracindo, R. (2009). O gestor escolar e as demandas da gestão democrática: exigencias, práticas, perfil e formação. Revista Retratos da Escola, 3(4), 135-147. http://retratosdaescola.emnuvens.com.br/rde/article/ view/107

López, L. (2016). Inteligencia emocional como herramienta que mejore las relaciones interpersonales de los estudiantes del 1er año sección "I" de la Unidad Educativa "Alejo Zuloaga. Trabajo de Grado de Especialista en Procesos Didácticos para el nivel Básico, no publicado. Universidad Pedagógica Experimental Libertador Instituto de Mejoramiento Profesional del Magisterio

López, L. (2016). Inteligencia emocional como herramienta que mejore las relaciones interpersonales de los estudiantes del 1er año sección "l” de la Unidad Educativa "Alejo Zuloaga. [Trabajo de Grado, Universidad Pedagógica Experimental Libertador] No publicado.

Luck, H. (2010). Gestão escolar: democracia e qualidade do ensino. Editorial Ática.

Machado, M. (2000). Desafios a serem enfrentados na capacitação de gestores escolares. Em Aberto, 17(72). 
http://rbep.inep.gov.br/ojs3/index.php/emaberto/article/view/2640

Miguel, F. (2020). Psicologia das emoções: uma proposta integrativa para compreender a expressão emocional. Psico-USF, Itatiba, 20(1), 153-162. https://doi.org/10.1590/1413-82712015200114

Sánchez, F. (2019). Fundamentos epistémicos de la investigación cualitativa y cuantitativa: Consensos y disensos. Revista Digital Investigación y Docencia, 13(1). 101-122. http://www.scielo.org.pe/scielo.php?scrip$\mathrm{t=sci}$ arttext\&pid=S2223-25162019000100008 
Copyright (c) 2021 Ramón Antonio Hernández Chirinos de Jesus y Francisco das Chagas Silva de Jesus Hernández

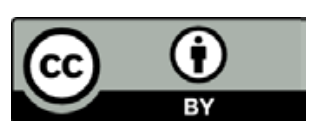

Este texto está protegido bajo una licencia internacional Creative Commons 4.0.

Usted es libre para Compartir-copiar y redistribuir el material en cualquier medio o formato - y Adaptar el documento - remezclar, transformar y crear a partir del material-para cualquier propósito, incluso para fines comerciales, siempre que cumpla las condiciones de Atribución. Usted debe dar crédito a la obra original de manera adecuada, proporcionar un enlace a la licencia, e indicar si se han realizado cambios. Puede hacerlo en cualquier forma razonable, pero no de forma tal que sugiera que tiene el apoyo del licenciante o lo recibe por el uso que hace de la obra.

\section{$\underline{\text { Resumen de licencia - Texto completo de la licencia }}$}

